\title{
Excrescent stops in American English
}

\author{
Cara Feldscher \& Karthik Durvasula*
}

\begin{abstract}
This study investigates whether excrescence is phonological epenthesis or articulatory overlap by investigating whether a prosodic domain boundary intervening between the nasal and the fricative affects insertion. Articulatory overlap effects are expected to remain constant across the board, whereas phonological epenthesis is expected to be targeted for domain-final lengthening. The duration results are that neither excrescence nor underlying segments are affected by domain-final lengthening, but excrescent closure is significantly shorter than underlying closure. The short segments support an articulatory overlap hypothesis, but the lack of final lengthening opens up new questions for further research.
\end{abstract}

Keywords. Domain-final lengthening; prosodic domains; excrescent stops; articulatory overlap; American English

1. Introduction. American English is frequently described as having an intrusive oral stop release between a nasal consonant and a voiceless fricative. A canonical example is "warmth", where /wo.m $+\theta$ / is pronounced [wo.mp $\theta]$. Other examples are "tenth" ([tent $\theta])$, "prince"

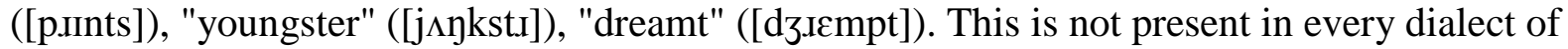
English, but commonly reported in American English, and seems to have little variation within dialects where it occurs (Fourakis and Port, 1986). There is some debate over whether this is an articulatory effect (Ohala and Ohala, 1993), or a phonological effect (possibly with articulatory motivation) (Clements and Hume, 1995; Naidoo, 2005; Iverson, 1989; among others). Analyses have been proposed using Optimality Theory, where the phonological motivation is articulatory ease, somewhat blurring these distinctions (Warner, 2002).

This experiment intends to probe whether this insertion is purely an effect of articulatory overlap or whether it is a phonological epenthesis by looking at sensitivity to prosodic domain final lengthening. Solé $(1992,1995)$ showed that allophonic nasalization and nasalization caused by gestural overlap react differently to changes in speech rate. I hypothesize that excrescence should also have different predicted behaviors if it were caused by epenthesis or articulatory overlap.

Section 2 reviews the previous literature on excrescence, including both proposed theoretical explanations and experimental research done. Some literature on separating phonetics from phonology is also reviewed, with background on final lengthening. Section 3 poses the research questions, and presents the hypotheses tested in this experiment. The experimental design is detailed in Section 4. Results are presented in Section 5 and further discussion continues in Section 6. Section 7 concludes.

2. Literature on excrescence. Much has been written about epenthesis in various languages and with various motivations, where epenthesis is typically argued to be a phonological solution to myriad problems such as vowel hiatus, prosodic boundary marking, or syllabic requirements.

\footnotetext{
* We wish to thank Yen-Hwei Lin, Alan Munn, and Marcin Morzycki, as well as the other members of the phonology group at MSU, and the audience at the 91st Annual Meeting of the Linguistic Society of America for valuable comments and discussion. Authors: Cara Feldscher, Michigan State University (feldsch3@msu.edu) \& Karthik Durvasula, Michigan State University (durvasul@gmail.com).
} 
Besides phonological epenthesis, there is also discussion of insertion with phonetic motivation, often referred to as "excrescent", "intrusive", or "emergent" segments in order to draw a distinction between these and epenthetic segments (Zygis, 2010). More narrowly, this study focuses on the oral release between a nasal and a voiceless fricative in American English, which also has been analysed as articulatory overlap and as phonological epenthesis in the literature. An overview of both articulatory overlap and phonological accounts will be given. A few production experiments that have been done on this subject are also summarized.

This is a wide-spread, but not ubiquitous, phenomenon, and similar effects have been discussed in other languages, some of which are referenced here. Studies of language change indicate that the insertion of a stop in this nasal-fricative environment, or similar environments such as after liquids, over time has occurred in multiple languages. For example, Ohala (1993) gives historic examples of excrescence between nasals and liquids: the Spanish word Alhambra resulting from the Arabic al hamra, and the French word chambre resulting from Latin camra. Synchronically, there are other examples from other languages present in the literature as well. For Valencian Catalan, Recasens (2012) details a perceptual study discovering intrusive stops to be perceived significantly less often than underlying stops. Warner and Weber (2001) report a similar finding for Dutch intrusive stops.

2.1. Phonetic motivation. Ohala (1993) explicitly declares the phenomenon of excrescent stops to be wrongly treated by some phonologists as epenthesis, whereas it should be classified as coarticulatory overlap. The orality is due to an early anticipatory raising of the velum, and the resulting stop is a natural result of the release of the closure mandated by a nasal stop. A very similar idea is repeated by Ohala and Ohala (1993), blaming the appearance of a homorganic stop on gestural overlap during the transition from a nasal consonant to an oral obstruent. This explanation says the oral release is a phonetically natural articulatory side effect of the transition from a nasal stop (with closure in the oral cavity) to a fricative (necessitating the release of the oral closure). This gestural overlap explanation partially accounts for why there is no equivalent insertion effect in a mirror-image environment of fricative-nasal. The fricative could bleed orality into the closure period, but there would be no release of oral constriction in the transition. So the mirror-image environment could naturally produce oral closure due to gestural overlap, but it could not produce a full oral closure plus release for an intrusive stop. This does not explain why an unreleased unaspirated stop is not produced in a fricative-nasal environment.

Additionally, such a gestural overlap would be equally possible when the segment after a nasal is not a voiceless fricative, and Ohala and Ohala (1993) do not limit it in such a way, referring to "the transition between a nasal consonant and a buccal obstruent". This either overpredicts the epenthesis or fails to specify why it is restricted within a language to certain subset environments to what is described. However, this idea of coarticulatory overlap is recurrent in all phonetic arguments for excrescent stops, and present in most phonological arguments as underlying motivation for epenthesis.

As this is not a language universal, this brings up the question of language-specific phonetics. While the distinction between phonetics and phonology has been thought of in the past as language-specific sound rules as opposed to universals about articulation and perception, studies have shown that both articulation and perception can vary by language. For example, Chitoran and Tiede (2013) found that effects from speech rate on articulatory reorganization were different between French and Georgian, in a way that reflected their phonotactics in addition to the gestures themselves. Shaw et. al. (2011) found that the timing of onset consonants differed between Moroccan Arabic and English, finding that it varied based on the syllable structure of the 
language, which indicates that there could still be some timing universals that appear languagespecific due to dependence on language-specific details like syllable structure. There may be more factors to gestural timing in a language that when accounted for make timing details wholly predictable, but at this point, no theory seems to exist that predicts which languages would have such an articulatory overlap and which would not.

2.2. Phonological EXPLANATIONS. Some theoretical takes on this phenomenon are occasionally wholly phonological, but others cite phonetic motivations as well. Some more major viewpoints on the issue start with the general idea of consonant epenthesis for syllabic well-formedness reasons, and others argue for a feature spreading solution, so those two viewpoints will be discussed here.

In their overarching discussion of epenthesis, Piggott and Singh (1985) touch on this particular case, considering it no different from other cases of phonological epenthesis. They offer the view that in a surface form, the sequence [ns] is blocked, and the solution of epenthesis results in [nts]. There is presumably no reason why this could not be expanded to better deal with the data by assuming a phonotactic constraint against sequences of nasals and voiceless fricatives in the same syllable in American English. However, one problem with this analysis is it requires special treatment of [s], treating [s] as a sonorant. They argue based on the patterning of [s] with sonorants in phonological rules in Old French (Old French Apocope, Cluster Simplification, and Early Syncope) that languages can treat [s] as a sonorant. To explain excrescence in American English, they suggest that American English is one such language. Given that this epenthesis occurs before other voiceless fricatives that lack extraordinary behavior like [s], this phonological motivation undergenerates and is insufficient to describe these intrusive stops.

Additionally, the motivation for consonant epenthesis is assumed to often be syllablestructure considerations, such as providing an onset or giving a stressed syllable a coda. However, consonant epenthesis to resolve illicit clusters instead of deletion or vowel epenthesis only increases the complexity of a consonant cluster in the syllable. English allows complex codas decreasing in sonority, so the new codas are perfectly licit, but still more marked than before the insertion. Thus reasoning following syllabic markedness reduction is difficult. For intrusive stops in Polish, Czaplicki (2010) discusses this point in order to specifically rebut the idea of this process as wholly phonological, comparing the motivated nature of consonant epenthesis when resolving vowel hiatus with epenthesis in this environment. A syllable-based theory of epenthesis with the standard view of markedness fails to produce epenthesis in this environment where it would only increase markedness, and so Czaplicki concludes this process to not be epenthesis. Instead, he argues that Ohala's claim of gestural overlap is correct, and that this effect begins as a phonetic effect. Starting from this gestural overlap, insertion over time of these segments, such as the examples from Ohala (1993), are described as phonological reanalysis resulting from perception of the phonetic oral release. The possibility of such a phonological reanalysis creates a possible confound in studies using existing monomorphemic words as test items for studying the synchronic phenomenon. They may be synchronically producing excrescent stops, or they may have been diachronically reanalyzed to have oral stops underlying there.

While also denying this to be a standard syllable-based consonant epenthesis, Wetzels (1985) cites phonological rule ordering as an argument that this stop still must be epenthesized phonologically, saying that it feeds word-final glottalization of stops, in that these excrescent stops are glottalized same as underlying stops when inserted in a cluster ending a word. However, word-final or phrase-final glottalization, in American English at least, is extensive although speakers may vary in their realization of it (Redi and Shattuck-Hufnagel, 2001). It would be in- 
teresting to see whether the phonetic realization of glottalization in excrescence closely mirrors that of underlying stops, or whether it more closely reflects phrase-final glottalization in general. The former would be in support of rule ordering as evidence for a phonological rule targeting an epenthetic segment, whereas the latter would not provide support for a phonological rule targeting an epenthetic segment.

Clements and Hume (1995) have one such analysis considering it synchronically a phonological process with phonetic motivation, arguing it to result from feature spreading in their model of feature geometry. (Other autosegmental solutions exist, but this one will be discussed as an exemplar of these theories. Differences in the feature geometric structures between theories still make the same predictions in terms of creating complex segments via node linking.) In their view, the epenthetic segment is a hybrid segment created from a link forming between the oral cavity node of the nasal and the root node of the following fricative. This phonological linking is realized as "a lag of the oral cavity constriction of the nasal or lateral into the following fricative". Thus the [-continuant] and place feature of the nasal and the [-nasal] feature of the fricative are combined in this intrusive segment to make an oral homorganic stop following the nasal. This intrusive stop, however, by remaining linked to the root node of the following fricative, is now part of a contour segment, the second half being the fricative. This is the explanation for the segment's shortened duration, the fact that it is essentially half of a segment rather than a whole insertion. This seems to make a prediction that the other half of the contour segment, the fricative, should also be shortened considerably in comparison to a full segment, which is not reported in the literature. In their explanation, this phenomenon is still described as having articulatory motivation for this feature spreading. Perhaps as a result of citing phonetic motivation, this analysis also leaves open the reasoning for why this excrescence is limited in environments to after nasals, or after laterals or other liquids in other languages. The phonetic analysis of Ohala and Ohala (1993) similarly overpredicted. One testable prediction this theory makes is that the second half of the contour segment, the following fricative, should also show reduced duration if it is half of a contour segment rather than a full segment.

Naidoo (2005) investigates an identical process in Zulu. The analysis follows the feature geometric perspective of Clements and Hume (1995), and also hypothesizes the results of an equivalent intrusive stop process to be affricates (stop+fricative). Given that Zulu has four affricate phonemes in addition to the relevant process of excrescence, the durations of underlying affricates and the ones created by the intrusive stop were compared. It is shown that the duration of underlying affricates is statistically longer than that of the ones created by excrescence, but most of the difference is in the closure duration. In American English, the intrusive stop is documented as being shorter than an underlying stop (Fourakis and Port, 1986; Ali et. al., 1979). Here Naidoo shows that the closure duration of the excrescence is also notably shorter than the closure duration of an underlying affricate in Zulu. Additionally, the following frication period in the insertion cases is shorter than the frication period of an underlying affricate. While the frication durational difference is reported to be statistically significant, it is shortened much less than the closure period is. This could be interpreted as showing that the created affricate is simply shorter overall than the underlying one, primarily in the closure period. This is the explanation given by Naidoo, who then introduces duration into the feature geometry to account for such a distinction. Or, these results could be unrelated to any link between the two segments; Fourakis and Port (1986) found that the overall word duration remained constant in a word with excrescence due to a slight shortening of the other segments. Impressionistically, the spectrograms shown by Naidoo for underlying affricates looked to be split somewhat evenly between closure 
and release. The split among the created affricates was impressionistically dissimilar. This could simply be a function of these "created affricates", or it could be interpreted as the two halves being durationally disparate because they are not two halves of the same segment, not linked in affrication as suggested by Clements and Hume (1995) and other feature geometric models.

2.3. Previous eXPeriments. Ali et. al. (1979) study this phenomenon, reporting on the presence of "silent gaps" in the spectrogram for the intrusive stop for multiple places of articulation, rather than only for coronals. They find that a period of oral closure is fairly regular, and that it may occur with or without an oral burst. In addition to measuring the oral closure, they report a shorter fricative in some cases, but without details of whether the fricative is short in comparison to a normal duration, or only in comparison to the other segments. They also speculate that the presence of a syllable or morpheme juncture between the nasal and the fricative might have led to exaggerated closure in the intrusive stops. In monomorphemic cases, however, a difficulty arises of determining whether or not the stop has been reanalyzed as underlying. For example, as previously mentioned, Ohala (1993) cites examples of language change where an intrusive stop is reanalyzed as underlying in the word, such as the evolution of French chambre from Latin camra.

Fourakis and Port (1986) have perhaps the most cited experimental study of these intrusive stops, documenting them in two dialects of English and comparing them to underlying stops in an equivalent position. This study compared multiple factors, including the durations of underlying and intrusive stops in the same environment. They found that in American English, between a nasal stop or lateral and a voiceless fricative there was a stop epenthesized $100 \%$ of the time. Before a voiced fricative, not only was a stop rarely epenthesized, but underlying stops were occasionally deleted, more often after a nasal and rarely after a lateral. In contrast, the speakers of South African English never epenthesized and never deleted between nasal stops or laterals and fricatives. Additionally, they found the inserted stop to be significantly shorter in duration than comparable underlying stops. The test items were all only one syllable words, and only coronals were tested.

Shosted (2011) ran an experiment considering effects of stress on intrusive stop formation by comparing epenthesis in stressed and unstressed syllables. Shosted also examined the effects of focus on intrusive stops by having speakers emphasize otherwise homophonous pairs, by saying things like I said PRINTS, not prince. Measuring the durations of intrusive stops in comparison to underlying ones, Shosted found that the duration distinction between underlying and excrescent stops is maintained in focused contexts. The typical effect of stress lengthening the segments in that syllable carried over to the epenthetic segment, but again the duration distinction remained.

2.4. Separating Articulatory Overlap From Phonology. The boundary between phonetics and phonology is often blurred, but some work has been teasing the two apart based on crosslinguistic patterns. Comparing phonological vowel nasalization in American English and allophonic nasalization in Spanish, Solé $(1992,1995)$ found that speech rate affected both differently. Phonological nasalization remained on a proportional amount of the vowel at different speech rates, whereas in Spanish it remained on the same duration of the vowel, as an articulatory overlap. A similar prediction can be made here that if excrescence is caused by an articulatory overlap, its duration should be unaffected by things that affect the duration of a phonological segment. An epenthetic segment, however, should be targetable for lengthening processes. 
The relevant lengthening process here is domain-final lengthening. Domain-final lengthening has been found to target both the stressed syllable in a word and the final syllable, specifically targeting the rime (Turk and Shattuck-Hufnagel, 2007). Additionally, the effect is cumulative, so larger boundaries should experience greater lengthening (Wightman et. al., 1992).

3. Research questions and predictions. The end goal of this study was to consider another aspect on the issue of whether this intrusive stop is a language-specific articulatory overlap effect, or something phonological like the epenthesis of a segment. This phenomenon has been examined with respect to several variables in other studies, as detailed in the previous section, but one factor that has not been considered is whether excrescence is sensitive to lengthening processes, as in domain-final lengthening. As referenced in the previous section, different predictions are made for articulatory overlap and for epenthesis.

The primary purpose is to investigate the effect of prosodic boundary at the location of the expected intrusive stop. As domain-final lengthening targets the phonological structure of the coda, increasing excrescence length at the boundaries of larger prosodic domains is in support of a phonological epenthesis analysis. An epenthetic segment should be syllabified into the coda which is targeted for final lengthening, whereas articulatory overlap should not be, as the duration of gesture does not affect articulatory overlap (Solé, 1992, 1995). Therefore, unchanged excrescence length at different boundary sizes is in support of an articulatory overlap analysis, as articulatory overlap should be invisible to a phonological process targeting codas.

A few other factors which have already been tested in the literature may be considered in favor of an articulatory overlap analysis. This study aims to replicate those in addition to investigating the effect of final lengthening. To begin with, an excrescence duration equivalent to that of an underlying stop would be a good indication of a full insertion. A notably shorter duration, paired with a shortened duration of an adjacent segment would support hypotheses of the formation of a contour segment. A shortened duration for the inserted segment paired with unaffected durations for the other segments, however, points toward the hypothesis of an articulatory overlap. Previous studies have noted a shorter duration of the excrescent stop. This intrusive stop appears after labial, coronal, and dorsal nasal stops (Ali et. al., 1979). This is not decisive between a phonological epenthesis or an articulatory overlap explanation, as it operates on a natural class, and the transitions from all of the nasal stops will involve the potential for overlap and creating an oral closure period.

\section{Experimental design.}

4.1. Methods. Ten speakers of American English from Michigan were recorded reading out the words or phrases in sentences, using PsychoPy (Peirce, 2007, 2009) to randomize the targets. For the first four participants, a Samson Meteor Mic was used to record on a Lenovo laptop. The last six were recorded on a Mac desktop with a Plantronics Audio 355 headset microphone. Participants were explicitly instructed to read them aloud, speaking casually and normally. All participants were recruited among Michigan State University undergraduate students and compensated with extra credit in an introductory linguistics course. They were asked before the experiment about native language in order to confirm that they speak the correct dialect, and data for non-Michigan or nonnative speakers was excluded.

Measurements were extracted in Praat (Boersma and Weenik, 2017). Annotation was done by hand, and then duration measurements were automated from there via a script. For annotating the excrescent stop and corresponding underlying stop, the nonnasal closures were marked and measured; aspiration was not included in the measurement in case of difficulty distinguishing it 
from the following fricative. The onset of oral closure was marked by the sudden weakening of formants and decline in the waveform, and the end was marked by the spike in the waveform and beginning of noise in the spectrogram that marks the aspiration or following fricative. An example is shown for an intrusive [p] in the word "himself", in Figure 1 below. These measurements were taken for the excrescent consonants and for the underlying ones in equivalent environments as a control group. The duration of the preceding nasal and following fricative were noted in the same manner. The nasal duration was marked primarily by the existence of antiformants, and secondarily by a flat waveform shape. The fricative was marked by the high-frequency energy on the spectrogram.

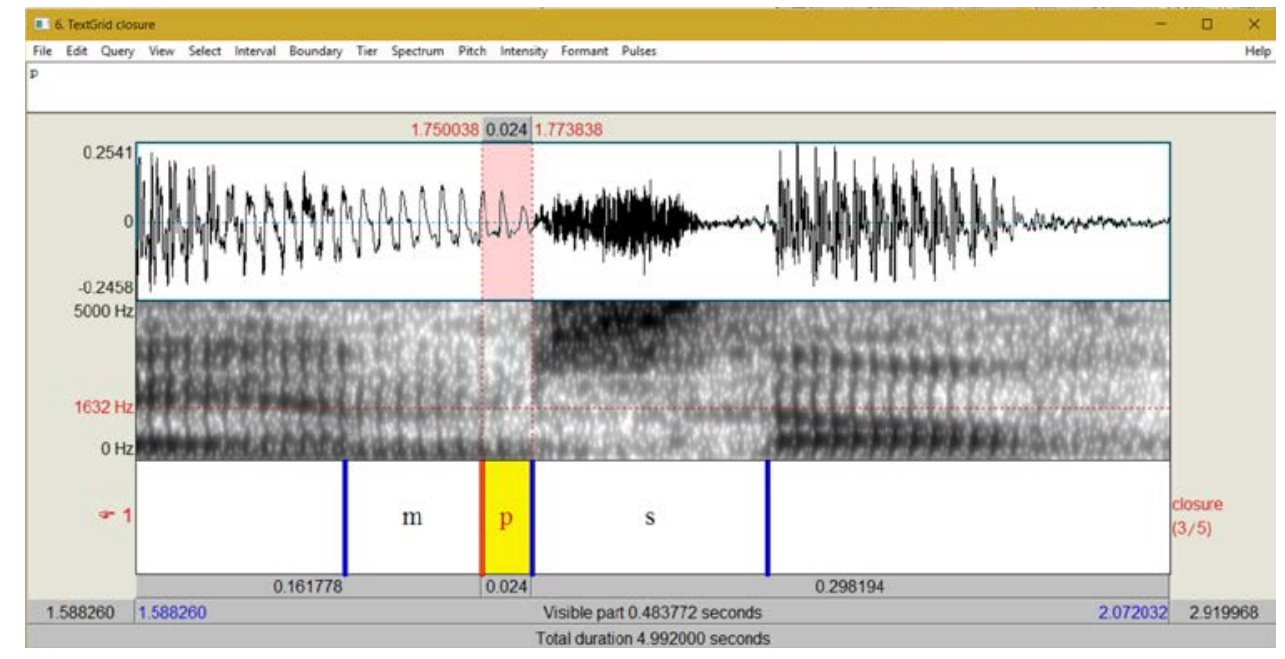

Figure 1. Screenshot of an excrescent stop in "himself".

The analysis was done in R (R Development Core Team, 2008). Data was discarded if there were speech errors causing the deletion of either the nasal or fricative in that sentence (357 out of 3600 tokens removed). Additionally, the data for each speaker was separately grouped, and the data was discarded for sentences with any measurements outside 2.5 standard deviations (32 out of 3243 remaining tokens removed). This was to eliminate speech errors causing slowing or pausing while controlling for individual speaker's speech rates.

Post hoc, in an attempt to further control for natural speech, these sentences were given to 10 different native speakers of Michigan English, recruited in an identical fashion, and participants were asked to rate each sentence as "natural" or not. Sentences with $>70 \%$ "natural" ratings were removed as well, given the possibility that odd or confusing syntax or semantic could affect fluency or naturalness of reading (530 out of 3211 remaining tokens removed).

Rather than include fillers, naturalistic sentences were created, with limited repetition in lexical items or sentence structure to avoid cluing participants in to the study's purpose. The target item location varied in the sentence, which is discussed further in the following section. Half the time the target sequence was the first or second word in, and the rest of the time, the target sequence was roughly four words in. There was a short, five sentence practice session of reading five other sentences before the experiment, followed by a chance to ask the experimenter any questions before starting the actual experiment. There were 60 sentences in the test portion, which the participants read in a randomized order twice, for an experiment run time of 12 minutes. This was a within-subjects design, and all speakers read all of the sentences for each condition. 
4.2. TARGET SEGMENTS AND ENVIRONMENT. Both voiceless and voiced fricatives followed nasals in the test items, and underlying voiceless and voiced stops were measured to compare against the excrescent stop durations. Voicing is a variable Fourakis and Port (1986) investigated in terms of the voicing of the following fricative, and it was not expected that excrescence would regularly appear in that environment.

For place of articulation, coronal, labial, and dorsal test items were all included. Most previous studies, such as Fourakis and Port (1986), Yoo and Blankenship (2003) and Shosted (2011) have focused on the alveolar [t], although Ali at. al., (1979) investigated the effect of different places on excrescence and found there to be none, so it was not expected that there would be an effect of POA on excrescence. Alveolar [t] is subject to a fairly high degree of word or phrasefinal glottalization, which has the potential to impede measurements (Redi and ShattuckHufnagel, 2001). In the case of velar nasals, a potential confound exists in that one could claim that velar nasals are underlyingly a nasal-stop sequence /ng/. Finally, a problem with labials and velars is that no underlying sequences of nasal-voiced stop are possible to include for comparison, as they are not licit in American English word-finally. In order to compensate for these potential issues as best as possible, all three places of articulation were included wherever possible, and the results were generalized over them.

Nasal+Fricative and Nasal+Stop+Fricative environments were not tested within a morpheme, due to an inability to tell what is underlying without making assumptions based on spelling. Instead, environments included across Compound word boundaries, across Verb-Object word boundaries, and across Subject-Verb word boundaries, in order to test the effect of different levels of prosodic boundaries predicted from the syntax of a sentence. The two halves of a compound are different Phonological Words (PW), so the compound-medial position was at a PW boundary (Jensen, 2004). The VO condition was across a Phonological Phrase (PhP) boundary, and the SV condition was also across a PhP boundary, as PhP domains begin at the left edge of each lexical item (Nespor and Vogel , 1986). The SV position is also across a Intermediate/Major Phrase (MajP) boundary in the intonational theory by Beckman and Pierrehumbert (1986), where the subject is a MajP due to being the highest phrase within the spellout domain (Kratzer and Selkirk, 2007). Although this is not a distinction that Nespor and Vogel (1986) make, the SV position is across a higher domain boundary than VO in Kratzer and Selkirk's (2007) theory, where phase boundaries in syntax predict the MajP domains. So SV can be considered minimally a PhP boundary in a theory of prosodic domains that builds from lexical items or the higher MajP boundary in a theory of prosodic domains that builds from phase based spellout as well.

If the following fricative is homorganic to the expected stop, the release of the stop may be difficult to distinguish. Therefore, no effort was made to prevent homorganic sequences in the stimuli and only the nonnasal closure was measured for the excrescent and underlying stops. The preceding vowel in all the test items was lax, so as to avoid any length differences on the consonants following tense or lax vowels. The excrescence duration measured was compared with that of underlying stops in equivalent environments.

5. Results. There were two main results. The first is that comparing the length of excrescent stops to underlying, excrescent stops were robustly shorter in all conditions, as is visible in Figure 2. A within-subjects two-factor ANOVA was run, with the independent factors being Closure Type (Excrescent or Underlying) and DOMAIN SIZE (Compound/PW, VO/PhP, SV/MajP), and the dependent variable being CODA DURATION. Only a significant main effect of 
ClOSURE TyPE was found $[\mathrm{F}(1,54)=52.85, \mathrm{p}<.001]$. This shorter duration has been reported in previous studies, and was expected in this experiment.

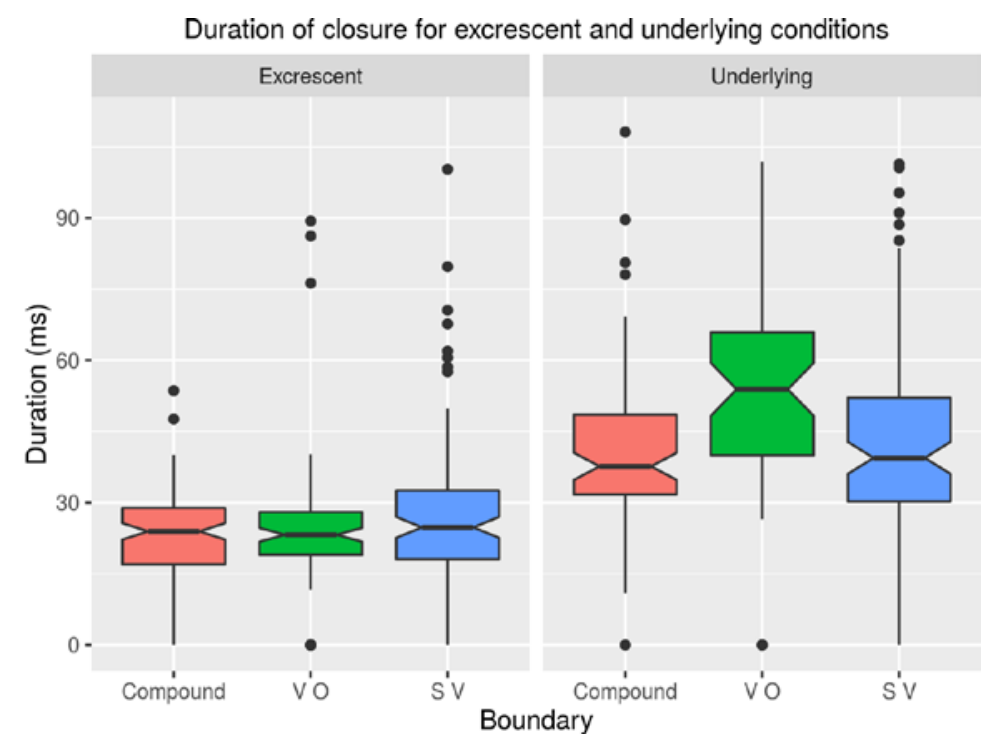

Figure 2. Bar plot comparing excrescent and underlying closure durations.

Excrescent stops were also omitted at a greater rate than underlying stops were deleted (Figure 3). The rate of zero excrescences does not change between Compound and VO positions, and while the rate of zeros increases between VO and SV positions, a one-way within-subjects ANOVA indicated no significant difference in percent of zero excrescences across boundary conditions $[\mathrm{F}(2,27)=.357, \mathrm{p}=.702]$. Therefore we can conclude that it's not a process bounded by either of the prosodic domains here. This analysis was carried out only for the excrescent condition, and rate of underlying stop deletion was not investigated.

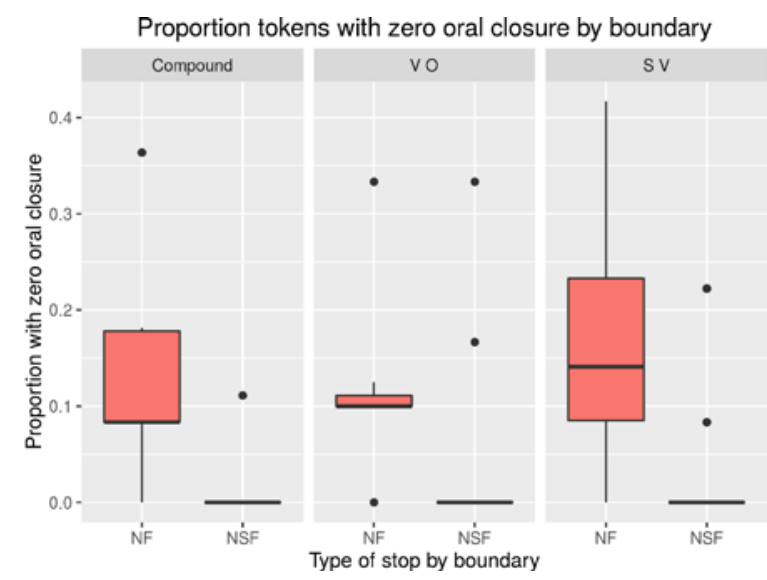

Figure 3. Bar plot showing rate of zero oral closures.

Nasals in the excrescence condition were longer than in the underlying condition (Figure 4). The shorter duration in the condition with the underlying stop can be explained as the segments in the coda compressing due to it being a complex coda. 


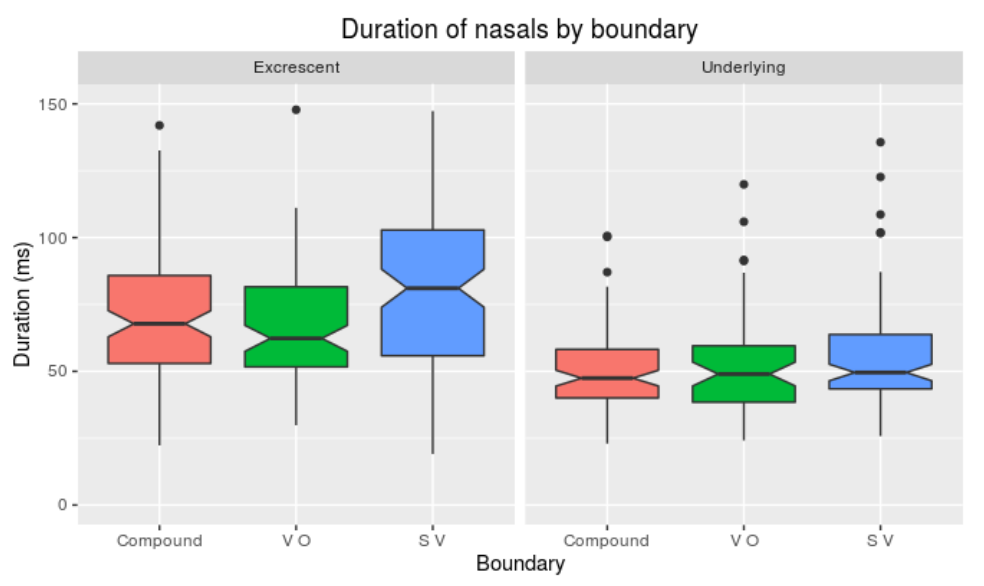

Figure 4. Bar plot comparing coda nasal durations.

The fricatives were measured to test a phonological analysis of excrescent stops as forming half of a contour segment with the following fricative, with the hypothesis that in that case, both the stop and fricative would show shortened duration. However, as can be seen in Figure 5, the fricative lengths seemed to vary greatly, but were not robustly shorter in the excrescence condition than in the underlying stop condition. In the excrescent condition, fricatives do pattern longer at the Phonological Phrase boundary than at the Phonological Word boundary, although they were not longer at the Major Phrase boundary than at the PhP boundary. This is not evidence of domain final lengthening, as the fricative was in the following onset and began the following prosodic domain. It could instead be domain-initial strengthening, although there is a large amount of variation, especially at the PhP boundary. Further study is needed to determine whether there is any robust pattern, and to determine what the source of any irregularities might be.

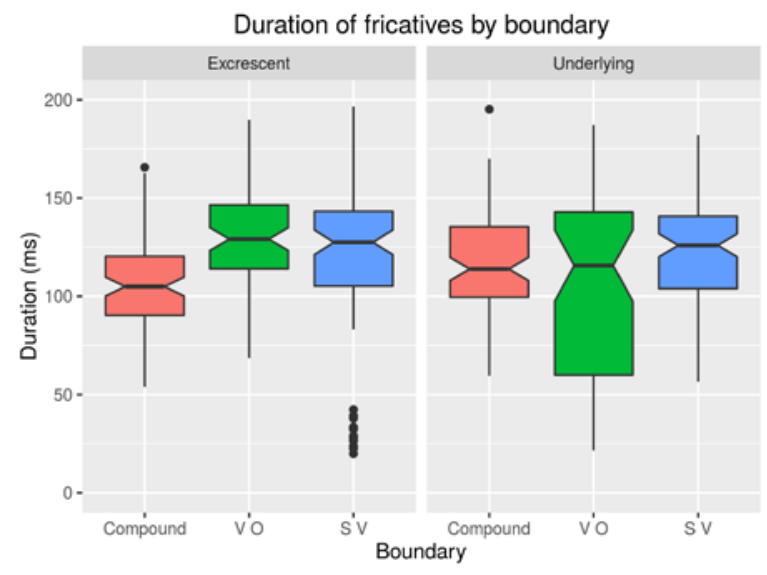

Figure 5. Bar plot of fricative durations.

The other result is that the duration of excrescence did not vary in accord with the prosodic domain size. However, as can also be seen in Figure 2, neither did the duration of the underlying stops. The previously mentioned two-factor ANOVA did not indicate a significant effect of DOMAIN BOUNDARY $[\mathrm{F}(2,54)=1.03, \mathrm{p}>.05]$. An odd result can be seen where the underlying stop is longer at VO boundaries than at Compound or SV boundaries. This is odd, because if VO and SV positions are both across a Phonological Phrase boundary, they are not predicted to pattern 
differently. If the SV positions were additionally across a larger MajP boundary, as Kratzer and Selkirk (2007) predict them to be, then it is expected that SV would show a greater effect of final lengthening than VO would. Greater lengthening at the VO position, compared to the SV position, is not predicted by any theory.

To further investigate the appearance of final lengthening in the underlying condition, Figure 6 shows the duration of underlying coda nasals next to the underlying stops. Underlying coda nasals also show no effect of domain-final lengthening, but pattern more uniformly than the stops do. The VO nasals differ from the VO stops in that they don't average longer than the other conditions, but rather are on par with the other two conditions. If the longer VO stop condition were showing domain-final lengthening, then the question would arise as to why it doesn't show up in the SV stop conditions or any of the nasal conditions. It makes for fewer unexplainable exceptions to say that neither coda stops nor nasals appear to be targeted for a cumulative domain-final lengthening process getting longer segments at the end of larger prosodic domains, leaving only the stop VO case as an open question.

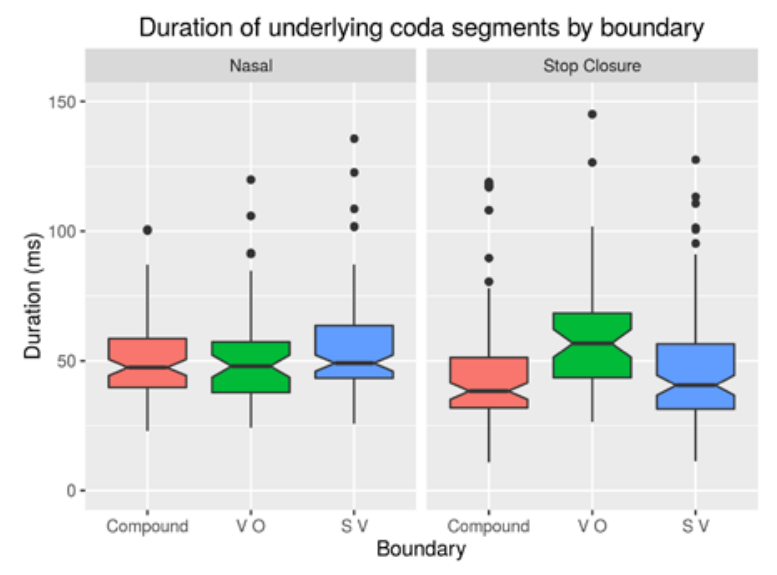

Figure 6. Bar plot of coda segment durations.

Finally, Figure 7 shows the overall coda durations for underlying Nasal+Stop+Fricative sequences. The VO codas are longest again, likely as a result of the coda stop durations. In addition to showing no evidence for domain-final lengthening cumulatively targeting the coda segments, the experiment results do not show evidence for cumulative domain-final lengthening on the overall coda duration, as the longest codas are not at the largest domain boundary.

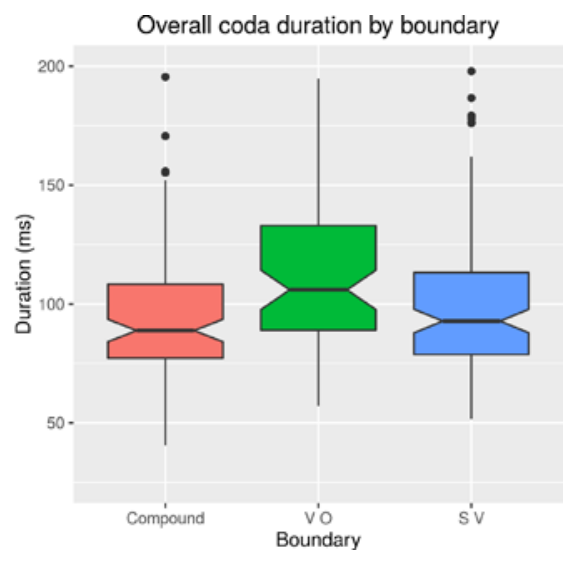

Figure 7. Coda durations. 


\section{Discussion.}

6.1. ClOSURE DURATION RESUlTS. The first finding was the predicted shorter closure for excrescent stops than underlying ones. This is in support of an articulatory overlap hypothesis. As previously discussed, this can be a language-specific gestural timing, explaining its presence in some languages and dialects, but not universally.

6.2. DomAin-FinAl LENGTHENING RESUlTS. The second finding was that the duration of excrescence did not vary according to domain size, but neither did the duration of the underlying stops. This is surprising, as domain-final lengthening has been reported multiple times in the literature. The hypothesis about whether excrescence is sensitive to lengthening depended on the assumption that phonological (coda) segments are sensitive to lengthening. As the underlying stops and overall coda underwent no lengthening, no new conclusion about excrescent stops can be drawn based on this pattern.

The new question emerges of why did final lengthening not appear in this study, yet appear in many others? There are a few possibilities. While final lengthening is reported in American English (Wightman et. al., 1992; Byrd, 2000), Hebrew (Berkovits, 1994), Dutch (CambierLangeveld et. al., 1997), Swedish (Horne et. al., 1995), and other languages, it might be subject to language variation. Michigan English might simply be a dialect without domain-final lengthening.

Another possibility relates to the particular domain boundaries investigated. This study looked at domains below the Intonational Phrase, meaning it is possible that lengthening occurs, but only at IP or higher boundaries. Cambier-Langeveld et. al. (1997) looked at lengthening in Dutch at domain boundaries and found that there was no difference between $\mathrm{PW}$ and $\mathrm{PhP}$, but both were shorter than Intonational Phrase (IP) and the Utterance Phrase (U). However, Wightman et. al. (1992) reports that lengthening is present at all levels, to a greater degree at higher levels. The latter study relied on break indices rather than syntactic cues to domain boundaries, which brings up the third possibility. Break indices are perceptual measures of prosodic distance, cued partially by word boundary or boundary tones, but also cued by pauses.

A third different but related possibility is that these conflicting results come from differing experimental designs. This experiment assumed the prosodic domains outlined by Nespor and Vogel (1986), as opposed to perceptual boundaries such as break indices. Cambier-Langeveld et. al. (1997) also used prosodic boundaries, but only found increased length at IP and U boundaries, the boundaries of both of which are strongly correlated with pauses. Other studies focused on perceptual boundaries and formulated experimental designs specifically to create pauses, in order to use break indices as a measure of prosodic domain boundary. In some cases, nonsense phrases designed to have an intonational contour and pauses were used (Fougeron and Keating, 1997). Edwards et. al. (1991) compared the same phonological string at positions like vocative or lists, which should be separate Intonational Phrase domains, set off by pauses, in comparison to within a word and word-final contexts. In other cases, participants were specifically told to pay careful attention to punctuation (Krivokapić and Byrd, 2012). Others used normal sentences, but created intonational contours other ways. For example, Price et. al. (1991) had newscasters read ambiguous sentences with disambiguating prosody. In all of these cases, the unifying element could be pauses. In the experiment here, there were no structures designed to create pauses, participants were instructed to read casually and naturally, and the data was discarded for any sentences where pauses created a duration over 2.5 standard deviations.

Other studies showing word-final lengthening could also possibly be attributed to pre-pausal lengthening instead of a boundary-final lengthening if they use carrier phrases. For example, 
Gordon and Munro (2007) compares word-medial, word-final, phrase-final, and utterance-final positions, finding that word-final is longer than word-medial, and phrase and utterance-final are longer than word-final. However, the production study relied on a handful of semantically neutral carrier phrases. Carrier phrases and other repetitive productions tend to receive an unnatural intonation, which occasionally sets off the test item (the item the speakers can tell is changing) with pauses or separate intonational contours.

6.3. FutURE DiRECTIONS. As discussed, there are multiple possibilities as to why there was no evidence of domain-final lengthening in these results. A study to tease those apart is currently underway with more Michigan English speakers. It has two blocks of sentences: first a block of naturalistic sentences designed to allow pauseless readings, followed by a block of monotonous carrier phrase sentences designed to produce pauses. For example, to test the PW boundary, the sentence "I will see the team play" will be repeated with different test items or fillers in the blank. Repetitions of the same sentence should create a carrier phrase intonation, and likely separate the test item from the rest of the sentence with pauses. The sentences have a target nasal or fricative simplex coda at the end of multiple boundaries (PW, PhP, IP, U). Given that IP and $\mathrm{U}$ are correlated with following pauses, they might not be directly comparable to PW and $\mathrm{PhP}$. So coda durations will be compared across different domain boundary levels for sentences produced without pauses, to see if the lengthening is correlated with domain size, and coda durations will be compared by length of the following pause for the sentences produced with pauses, to see if lengthening is correlated with pause duration instead.

If final lengthening is actually pre-pausal lengthening, the coda durations are not predicted to increase with boundary size in sentences without pauses, but rather coda durations are predicted to increase when followed by longer pauses. If final lengthening is not pre-pausal, but rather correlates with higher domains, greater coda duration is predicted in sentences where the target item appears at the end of larger domains. Finally, if final lengthening did not appear in this study because it is dialectal, no lengthening distinction is predicted to appear in this follow-up experiment, as the speakers are also Michigan English speakers.

As for the study of excrescent stops, this study could not weigh in on whether they are affected by lengthening phenomena that target phonological segments. The just-described experiment could better isolate what environment should be targeted by final lengthening. If it turns out that lengthening is pre-pausal instead, then this experiment could be rerun with environments that produce excrescence before pauses instead of before prosodic domain boundaries. This means that none of the Nasal+Fricative sequences would be able to be across word boundaries, limiting potential environments to within or across morphemes. A better idea might to find another environment targeting phonological structures for lengthening, or other phenomena.

7. Conclusion. Here a study was presented that attempted to answer the question of whether excrescent stops in American English are phonological epenthesis or articulatory overlap. Previous studies have investigated this, and leaned toward the articulatory overlap hypothesis due to the shortened duration of excrescent stops when compared with underlying ones in the same position. The novel contribution of this experiment aimed to test excrescence with regards to domain-final lengthening, with the hypothesis that a phonological segment should be targetable for domain-final lengthening, but articulatory overlap should not be. Crucially, the experimental design used naturalistic sentences incorporating the Nasal-Fricative sequence across multiple prosodic domain boundaries using the boundaries from Nespor and Vogel (1986).

The durational findings supported previous experimental findings that excrescence is reliably shorter than underlying stops, which suggests an articulatory overlap analysis. However, no 
evidence of domain-final lengthening surfaced in the data recorded here for excrescent stops, underlying stops, or codas as a whole. The lack of domain-final lengthening in the control group of underlying segments is a problem with the premise of any hypothesis about whether excrescence is sensitive to domain-final lengthening. No conclusion can be drawn about the nature of this excrescence from their lack of domain-final lengthening in this data, as a result. Instead, a new question emerged about why no evidence of domain-final lengthening was found here when it has been claimed to be repeatedly attested in the literature.

\section{References}

Ali, L., Daniloff, R., and Hammarberg, R. (1979). Intrusive stops in nasal-fricative clusters: an aerodynamic and acoustic investigation. Phonetica, 36(2):85-97.

Beckman, M. E. and Pierrehumbert, J. B. (1986). Intonational structure in Japanese and English. Phonology, 3(01):255-309.

Berkovits, R. (1994). Durational effects in final lengthening, gapping, and contrastive stress. Language and Speech, 37(3):237-250.

Boersma, Paul \& Weenink, David (2017). Praat: doing phonetics by computer [Computer program]. Version 6.0.29, retrieved 24 May 2017 from http://www.praat.org/.

Byrd, D. (2000). Articulatory vowel lengthening and coordination at phrasal junctures. Phonetica, 57(1):3-16.

Cambier-Langeveld, G. et al. (1997). The domain of final lengthening in the production of Dutch.

Chitoran, I. and Tiede, M. (2013). Gestural reorganization under rate pressure interacts with learned language-specific phonotactics. In Proceedings of Meetings on Acoustics ICA2013, volume 19, page 060199. ASA.

Clements, G. N. and Hume, E. V. (1995). The internal organization of speech sounds.

Czaplicki, B. (2010). Emergent stops in English and in Polish: Against syllable-based accounts. Poznań Studies in Contemporary Linguistics, 46(2):177-191.

Edwards, J., Beckman, M. E., and Fletcher, J. (1991). The articulatory kinematics of final lengthening. The Journal of the Acoustical Society of America, 89(1):369-382.

Fougeron, C. and Keating, P. A. (1997). Articulatory strengthening at edges of prosodic domains. The Journal of the Acoustical Society of America, 101(6):3728-3740.

Fourakis, M. and Port, R. (1986). Stop epenthesis in English. Journal of Phonetics, 14(2):197221.

Gordon, M. and Munro, P. (2007). A phonetic study of final vowel lengthening in Chickasaw. International Journal of American Linguistics, 73(3):293-330.

Horne, M., Strangert, E., and Heldner, M. (1995). Prosodic boundary strength in Swedish: Final lengthening and silent interval duration. In Proceedings ICPhS, volume 95, pages 170-173.

Iverson, G. K. (1989). On the category supralaryngeal. Phonology, 6(02):285-303.

Jensen, J. T. (2004). Principles of generative phonology: an introduction, volume 250. John Benjamins Publishing.

Kratzer, A. and Selkirk, E. (2007). Phase theory and prosodic spellout: The case of verbs. The Linguistic Review, 24(2-3):93-135.

Krivokapić, J. and Byrd, D. (2012). Prosodic boundary strength: An articulatory and perceptual study. Journal of Phonetics, 40(3):430-442.

Naidoo, S. (2005). Intrusive stop formation in Zulu: An application of feature geometry theory. PhD thesis, Stellenbosch: University of Stellenbosch. 
Nespor, M. and Vogel, I. (1986). Prosodic phonology (studies in generative grammar 28). Dordrecht: Foris.

Ohala, J. and Ohala, M. (1993). The phonetics of nasal phonology: theorems and data. Phonetics and phonology, 5:225-249.

Ohala, J. J. (1993). Coarticulation and phonology. Language and speech, 36(2-3):155-170.

Peirce, J. (2007). Psychopy - psychophysics software in python. Journal of Neuroscienve Methods, 162:8-13.

Peirce, J. (2009). Generating stimuli for neuroscience using psychopy. Front. Neuroinform.

Piggott, G. L. and Singh, R. (1985). The phonology of epenthetic segments. Canadian Journal of Linguistics- Revue Canadienne de Linguistique, 30(4):415-451.

Price, P. J., Ostendorf, M., Shattuck-Hufnagel, S., and Fong, C. (1991). The use of prosody in syntactic disambiguation. the Journal of the Acoustical Society of America, 90(6):29562970.

R Development Core Team (2008). R: A language and environment for statistical computing. $\mathrm{R}$ Foundation for Statistical Computing, Vienna, Austria. ISBN 3-900051-07-0.

Recasens, D. (2012). The phonetic implementation of underlying and epenthetic stops in word final clusters in Valencian Catalan. Journal of the International Phonetic Association, 42(01):65-90.

Redi, L. and Shattuck-Hufnagel, S. (2001). Variation in the realization of glottalization in normal speakers. Journal of Phonetics, 29(4):407-429.

Shaw, J. A., Gafos, A. I., Hoole, P., and Zeroual, C. (2011). Dynamic invariance in the phonetic expression of syllable structure: a case study of Moroccan Arabic consonant clusters. Phonology, 28(03):455-490.

Shosted, R. K. (2011). An articulatory-aerodynamic approach to stop excrescence. Journal of Phonetics, 39(4):660-667.

Solé, M.-J. (1992). Phonetic and phonological processes: The case of nasalization. Language and Speech, 35(1-2):29-43.

Solé, M.-J. (1995). Spatio-temporal patterns of velopharyngeal action in phonetic and phonological nasalization. Language and Speech, 38(1):1-23.

Turk, A. E. and Shattuck-Hufnagel, S. (2007). Multiple targets of phrase-final lengthening in American English words. Journal of Phonetics, 35(4):445-472.

Warner, N. (2002). The phonology of epenthetic stops: implications for the phonetics-phonology interface in optimality theory. Linguistics, 40(1; ISSU 377):1-28.

Warner, N. and Weber, A. (2001). Perception of epenthetic stops. Journal of Phonetics, 29(1):53-87.

Wetzels, W. L. (1985). The historical phonology of intrusive stops-a nonlinear description. Canadian Journal of Linguistics-Revue Canadienne de Linguistique, 30(3):285-333.

Wightman, C. W., Shattuck-Hufnagel, S., Ostendorf, M., and Price, P. J. (1992). Segmental durations in the vicinity of prosodic phrase boundaries. The Journal of the Acoustical Society of America, 91(3):1707-1717.

Yoo, I. W. and Blankenship, B. (2003). Duration of epenthetic [t] in polysyllabic American English words. Journal of the International Phonetic Association, 33(02):153-164.

Zygis, M. (2010). Typology of consonantal insertions. ZASPiL Nr. 52-September 2010 Papers from the Linguistics Laboratory, page 111. 